\title{
Study on rheological properties of polymer grouting materials with different expansion rates
}

\author{
Zheng Zhigang, Liu Tao, Hong Shaoyou, Huang Zhi, Wu Yintan, Huang Hong \\ Shenzhen Yuetong Construction Engineering Co., Ltd
}

\begin{abstract}
Non-water reactive polymer grouting technology is an important means to control water-induced engineering disaster emergency rescue. However, the time-varying viscosity of polymer slurry and the expansion force of slurry reaction are the main factors affecting the grouting treatment effect. Therefore, it is necessary to study the time-varying viscosity of polymer slurry and the expansion force of slurry reaction. Firstly, the expansion rate of polymer slurry with different expansion ratio is tested and checked by the selfmade expansion rate measuring cylinder. Then, the reaction expansion force and viscosity of polymer slurry with different expansion ratio are tested and studied by the expansion force testing device and NDJ-5T digital rotational viscometer. The results show that the expansion rate of polymer slurry used in this paper is basically the same as the factory instructions At the same time, the viscosity of polymer slurry increases exponentially with time, and the curve is steeper, that is to say, the viscosity increases rapidly with time.
\end{abstract}

\section{Introduction}

China has a huge scale of infrastructure projects. There are many public facilities in service and under construction, such as roads, railways, airports, dams, reservoirs, etc., which are usually accompanied by engineering diseases. Most of them are caused by water, including road collapse, subsidence, water inrush and water gushing-out, leakage [1]. In view of the above diseases, Zhengzhou University has developed a complete set of high polymer grouting technology and equipment, which has shown good performance in emergency rescue, repair and protection of engineering diseases caused by water. Among them, Shi Mingsheng [2] first carried out a series of experiments on the mechanical properties, impermeability, durability and other engineering properties of high polymers, and put forward the theory of high polymer split grouting. The basic non-water reactive high polymer grouting technology studied in this paper has been developed and applied in various fields of foundation engineering. Guo Chengchao et al. [3-4], Shi Mingsheng et al. [5] successively applied the high polymer grouting technology to the repair of cement concrete pavement and tunnel diseases, and evaluated the effect of grouting repair with FWD, Ground Penetrating Radar and other advanced detection technologies. Later, Bian Xuecheng et al. [6] applied high polymer grouting technology to the repair of high-speed railway subgrade, Shi Mingsheng et al. [7] developed high polymer anchor and carried out experimental research on its bonding performance with silt, both of which proved the remarkable effect of polymer on the reinforcement and repair of engineering infrastructure. In recent years, the high polymer grouting technology has been gradually improved, successively developed the high polymer dam seepage prevention grouting technology [8], the semirigid base high polymer penetration grouting technology [9] and the hollow pipeline high polymer repair technology [10] However, Shi Mingsheng [2] once pointed out that the high polymer slurry has significant rheological property, that is, the time-varying viscosity of the slurry. Strengthening the research on the rheological property of the high polymer slurry will help to calculate the diffusion range of the slurry more accurately and lay a foundation for the development of high polymer grouting technology.

However, in the field of grouting engineering research, the research on slurry rheology is mainly based on cement slurry grouting materials. Among them, Ruan Wenjun [11] proved the existence of time-varying viscosity of cement slurry through a large number of experimental studies, and established the diffusion model of rock fracture grouting based on this. Li Shucai et al. [12], Liu Quansheng et al. [13] successively considered the influence of underground water, temperature and hydration time, and studied the timevarying viscosity of cement slurry. Liu Yu et al. [14] studied the rheological properties of cement-based materials, and introduced different rheological models for verification. Later, Song Guozhuang et al. [15], Zhang Xin et al. [16] explained the rheological mechanism of cement grouting materials from the microstructure, which provided theoretical guidance for cement grouting engineering. However, there are few reports on the rheological properties of high polymer grouting materials, but Li Xinfa et al. [17] have studied the rheological properties of modified high polymer

\footnotetext{
* Corresponding author: zgangz@tom.com
} 
waterproof materials through plate rheometer, which further proves that the high polymer has certain rheological properties, and provides a certain reference for the rheological properties of high polymer grouting materials. In addition, the literature [13] also points out that the rheological property of grouting material is an important basis for the study of grouting diffusion model, and the study of high polymer grouting material diffusion model has been done by Li Xiaolong et al. [1819], but the influence of high polymer rheological properties has been ignored. Therefore, the research on the rheological properties of high polymer grouting material has certain engineering value and theoretical significance. Therefore, according to the actual needs of the project, several kinds of high polymer grouting materials with different expansion ratio are tested for expansion force and viscosity in this paper, in order to provide a reference for the development and application of high polymer grouting technology.

\section{Verifying Test of High Polymer's Expansion Rate}

There are various kinds of high polymer materials commonly used in engineering, but few are more commonly used. Here, three kinds of polymer grouting materials commonly used in engineering (10 times expansion rate, 20 times expansion rate and 30 times expansion rate) are taken as the research object. Firstly, the expansion rate of ex-factory high polymer is calibrated and verified. According to the requirements of the test, the reaction vessel for high polymer expansion rate test is made by author himself as shown in Figure 1, which is mainly composed of plexiglass cylinder with bottom. Meanwhile, in order to observe the change of expansion volume, a scale is pasted on the reaction cylinder. In addition, the test was carried out at room temperature. The weight of the two-component high polymer grouting material with the same quality was $80 \mathrm{~g}$ after mixing, and the height was about $2.5 \mathrm{~cm}$ after pouring into the reaction vessel.

Fig. 1. and Fig. 2. show the morphological photos of the three kinds of high polymers' consolidated body in the reaction cylinder and the comparison diagram of the slurry height before and after the reaction. It can be seen from the figure that the three kinds of high polymers grouting materials expand to a certain extent after reaction, and Fig. 2 can clearly see that the three kinds of high polymers expand from the original height of $2.5 \mathrm{~cm}$ to $11.5 \mathrm{~cm}, 21 \mathrm{~cm}$ and $31.5 \mathrm{~cm}$. Among them, compared with 10 times expansion ratio polymer, the height of 20 times and 30 times expansion ratio polymer is 1.83 times and 2.74 times respectively. Within the allowable range of test error, it can be proved that the ex-factory expansion ratio of three kinds of high polymer is reliable.

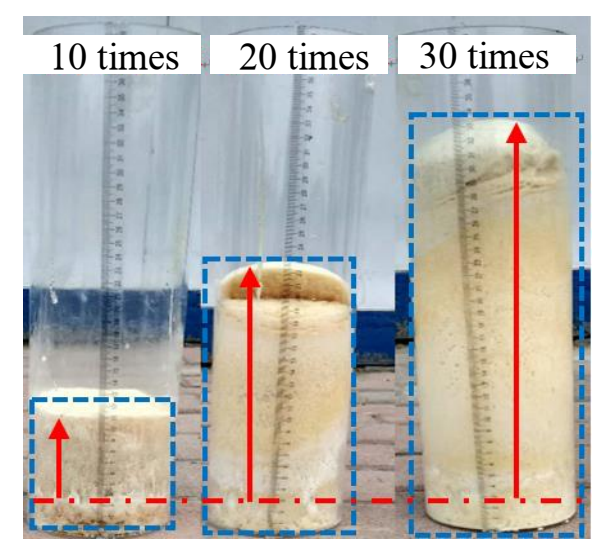

Fig. 1. Polymer consolidation after reaction.

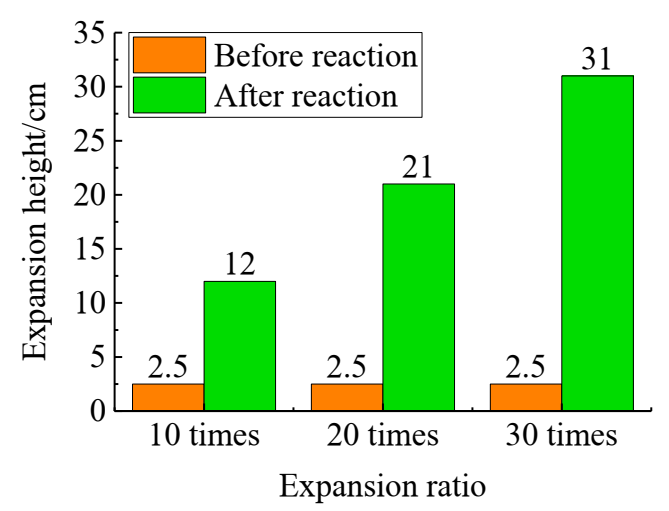

Fig. 2. Comparison of slurry height before and after reaction

\section{Experimental Test of High Polymer's Expansion Force}

In the literature[20], a series of experimental studies have been carried out on the expansion force of high polymer grouting materials, and the functional relationship formula between the density of high polymer consolidation and the maximum expansion force has been established, but the expansion force of polymer grouting materials with different expansion rates has not been analyzed. Here, the author designs the expansion force test device shown in Fig. 3., which is mainly composed of reaction cylinder, pressure sensor, data acquisition instrument, grouting head and exhaust hole. Through the pressure sensor and data acquisition instrument, the real-time measurement of slurry expansion force in the reaction cylinder is realized. In order to facilitate the analysis, this paper controls the same grouting amount to test the expansion force in three kinds of multiplying rates, and the test results are shown in Fig. 4. Obviously, the curve of expansion force with time for three kinds of high polymers grouting materials increases first and then tends to be stable, and the expansion force increases gently with time in the first $15 \mathrm{~s}$ of reaction, and increases rapidly in the period of $15 \mathrm{~s}-40 \mathrm{~s}$, and then keeps stable basically. At the same time, in the case of the same grouting amount, the higher the expansion ratio of high polymer, the greater the final stable value of expansion force, and the shorter the time 
to reach the stable value. For example, the final stable values of high polymer with 10 times, 20 times and 30 times expansion ratio are $1.05 \mathrm{MPa}, 1.55 \mathrm{MPa}$ and $2.2 \mathrm{MPa}$ respectively, while the corresponding time to reach the stable value is $30 \mathrm{~s}, 35 \mathrm{~s}$ and 40 s respectively. This shows that the expansion force generated by the high polymer slurry reaction is in direct proportion to the expansion rate of the high polymer itself, and it can also be confirmed from the pictures of the high polymer consolidators with different expansion ratios in Figure 1, that is, the higher the expansion rate of the high polymer, the more sufficient the slurry reaction, the more uniform the color of the consolidators, and the greater the expansion force generated in the corresponding reaction process.

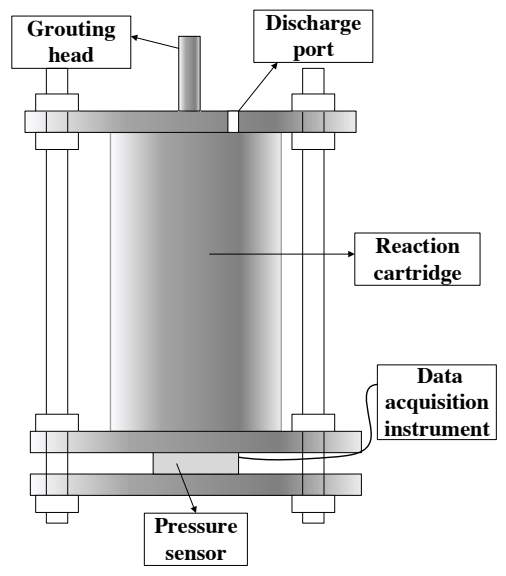

Fig. 3. Schematic diagram of polymer expansion force test device

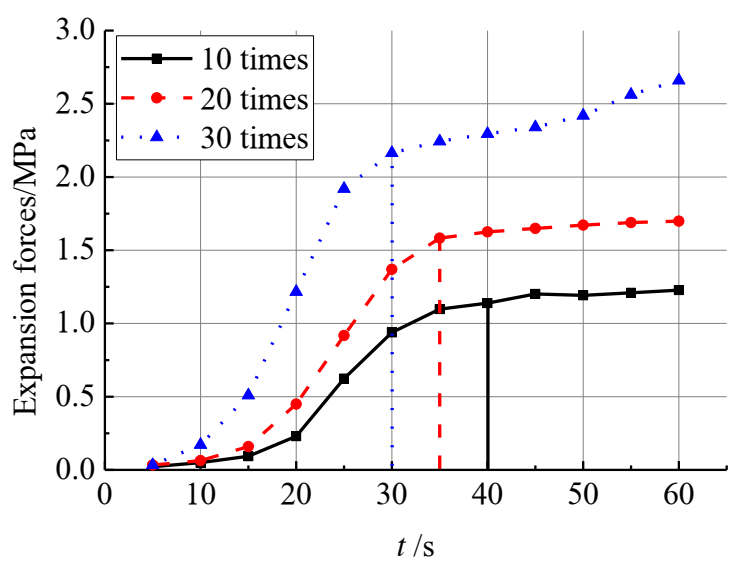

Fig. 4. Curve of expansion force of polymer with time

\section{Experimental Test of High Polymer's Expansion Force}

High polymer grouting materials have obvious rheological properties, that is, the time-varying viscosity of the slurry. Like the expansion force, the time-varying viscosity of high polymer slurry is also an important factor affecting the diffusion distance of slurry. Different from the cement slurry, the high polymer slurry has a faster reaction and shorter consolidation time, which is very suitable for engineering emergency rescue, and the time-varying viscosity of slurry has a decisive role in the grouting effect, so it is necessary to strengthen the research on the rheological properties of high polymer slurry. In this paper, the digital rotational viscometer shown in Fig. 5. is introduced to test the viscosity of three kinds of high polymer grouting materials, and the test results are shown in Fig. 6.. It can be seen from the figure that the viscosity of three kinds of high polymer slurry increases exponentially with time, and the higher the expansion rate is, the steeper the curve is, that is, the higher the expansion rate is, the shorter the consolidation time of the slurry reaction is. For example, the viscosity of high polymer slurry with 30 times expansion rate in Fig. 6. changes abruptly in $18 \mathrm{~s}-20$ s, which indicates that the high polymer slurry starts to change from liquid to solid in this period, and the viscosity of the slurry increases corresponding to the shear blockage of viscometer. The viscosity of high polymer slurry with 20 times and 30 times expansion rate also changes abruptly in $34 \mathrm{~s}-36 \mathrm{~s}$ and $46 \mathrm{~s}-48 \mathrm{~s}$ periods respectively.

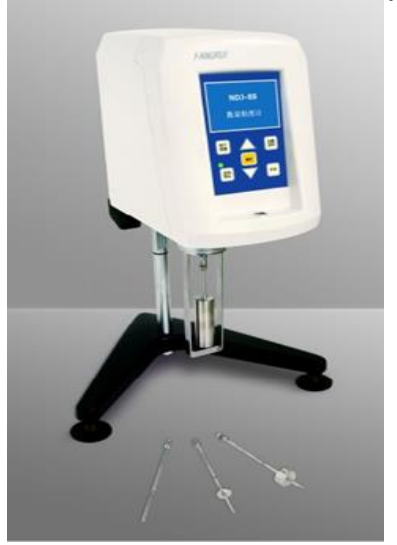

Fig. 5. NDJ-5T digital rotational viscometere

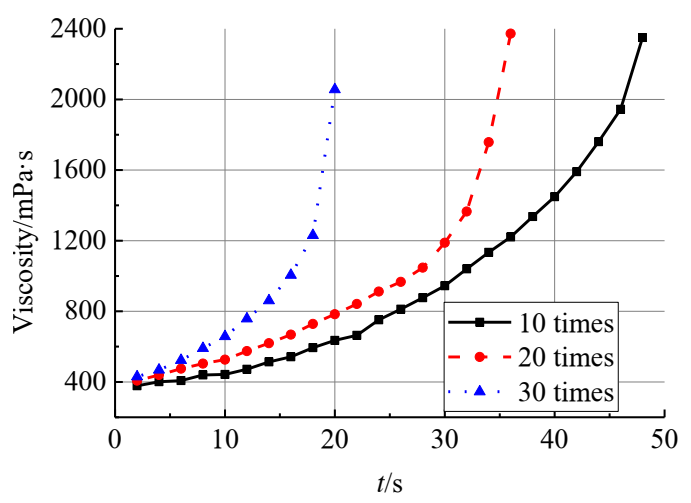

Fig. 6. Viscosity curve of polymer with time

In order to further study the rheological properties of high polymers, the test results in Fig. 6 are non-linear fitted, and the fitting formula is shown in formula (1-3), where $\eta$ is viscosity. From formula (1-3), it can be seen that with the increase of the expansion rate of high polymers, the constant terms of the index part of the formula increase gradually, which are $0.061,0.073$ and 0.143 respectively. This further confirms the above view that the higher the expansion rate of high polymer is, the larger the constant term of the index part of the formula is, and the steeper the viscosity curve of high polymer 
slurry is, the shorter the consolidation time of slurry is. Therefore, high polymer grouting materials can be selected reasonably according to the scope of reinforcement and rescue in order to achieve the optimization of grouting effect.

Viscosity with 10 times expansion rate-time relationship formula

$$
\eta=102.33 \mathrm{e}^{0.061 t}+271.27
$$

Viscosity with 20 times expansion rate-time relationship formula

$$
\eta=109.72 \mathrm{e}^{0.073 t}+294.36
$$

Viscosity with 30 times expansion rate-time relationship formula

$$
\eta=85.73 \mathrm{e}^{0.143 t}+316.34
$$

\section{Conclusions}

In this paper, the expansion force and viscosity of three kinds of high polymer grouting materials with different expansion rates are tested by a self-designed expansion force testing device and a numerical rotation viscometer, and the following conclusions are obtained:

(1) Three kinds of high polymer grouting materials and their expansion rates used in this paper are basically consistent with the factory calibration;

(2) The curve of expansion force with time for three kinds of high polymers grouting materials increases first and then tends to be stable, and the higher the expansion rate is, the greater the final stability value of the expansion force is, and the shorter the time to reach the stability value is;

(3) The viscosity of three kinds of high polymer slurry increases exponentially with time, and the higher the expansion rate is, the steeper the curve is, and the shorter the consolidation time is.

\section{References}

1. F. M. Wang, Y. F. Fan, C. C. Guo, JOHE, 37, 111(2018)

2. M. S. Shi, DUOT, (2011)

3. C. C. Guo, F. M. Wang, Y. H. Zhong, HIGHWAY, 232-236, (2008)

4. C. C. Guo, F. M. Wang, J. G. Xu, BATMACT, 25, 60-62, (2008)

5. M. S. Shi, X. Y. Ma, F. M. Wang, HHS, 28,74-77, (2010)

6. X. C. Bian, C. Cheng, F. M. Wang, J. Q. Jiang, Y. M. Chen, CJOGE, 36, 562-568, (2014)

7. M. S. Shi, W. Y. Xia, F. M. Wang, H. Liu, Y. H. Pan, CJOGE, 36, 724-730, (2014)

8. F. M. Wang, J. Li, M. S. Shi, C. C. Guo, JOHE, 35, 1-11, (2016)

9. F. M. Wang, W. H. Li, C. C. Guo, H. Y. Fang, C. Cui, JOBJTU, 43, 1-7, (2019)

10. B. Li, H. Y. Fang, F. M. Wang, JOZZU (Engineering Science), 40, 62-66, (2019)
11. W. J. Ruan, CJORMAE, 24, 2709-2714, (2005)

12. S. C. Li, W. W. Han, Q. S. Zhang, R. T. Liu, X. J. Wong, CJORMAE, 32, 1-7, (2013)

13. S. Q. Liu, C. B. Lu, B. Liu, X. L. Lu, CJORMAE, 33, 3730-3740, (2014)

14. Y. Liu, C. J. Shi, D. W. Jiao, X. P. An, JOTCCS, 45, 708-716, (2017)

15. G. Z. Song, L. J. Wang, Y. R. Zhang, Y. Guo, Y. P. Cao, JOHIOT, 50, 31-35, (2018)

16. X. Zhang, R. J. Qiu, S. P. Hou, Y. H. Wan, S. Wang, Concrete, 72-76+81, (2019)

17. X. F. Li, W. Li, N. Liu, S. L. Liu, Y. Zhang, PMSAE, 25, 115-117, (2009)

18. X. L. Li, T. T. Zhang, F. M. Wang, Y. H. Zhong, B. Zhang, RASM, 38, 3491-3497+3504, (2017)

19. X. L. Li, D. Jin, F. M. Wang, Y. H. Zhong, B. Zhang, CJORMAE, 37, 1207-1217, (2018)

20. Y. H. Pan, ZZU, (2008) 